\section{INFLAMMATORY COMPLICATIONS OF CGRP MONOCLONAL ANTIBODIES}

1,2,3 Jason C Ray, ${ }^{4,5}$ Penelope Allen, ${ }^{6}$ Ann Bacsi, ${ }^{3,7}$ Julian Bosco, ${ }^{2,3}$ Luke Chen, ${ }^{3,8}$ Michael Eller, ${ }^{4,5}$ Lyndell Lim, ${ }^{9}$ Hock Kua, ${ }^{10}$ Manjit Matharu, 2,3,11 Mastura Monif, 2,3 Richard Stark, ${ }^{2,3}$ Elspeth Hutton. 'Department of Neurology, Austin Health, Melbourne, VIC, Australia; ${ }^{2}$ Alfred Health, Melbourne, VIC, Australia; ${ }^{3}$ Monash University, Melbourne, VIC, Australia; ${ }^{4}$ Centre for Eye Research Australia, Royal Victorian Eye and Ear Hospital, East Melbourne, VIC, Australia; ${ }^{5}$ Department of Surgery, University of Melbourne, Parkville, VIC, Australia; ${ }^{6}$ Integrated Specialist Medical Care, Macquarie University Hospital and North Shore Private Hospital, Sydney, NSW, Australia; ' ${ }^{7}$ Allergy, Asthma and Clinical Immunology, Alfred Health, Melbourne, VIC, Australia; ${ }^{8}$ Department of Neurology, Monash Medical Centre, Melbourne, VIC, Australia; ${ }^{9}$ Department of Pathology, Monash Medical Centre, Melbourne, VIC, Australia; ${ }^{10}$ University College London (UCL) Queen Square Institute of Neurology and The National Hospital for Neurology and Neurosurgery, Queen Square, London, UK; ${ }^{11}$ Department of Neurology, Royal Melbourne Hospital, Melbourne, VIC, Australia

\subsection{6/bmino-2021-ANZAN.76}

Background CGRP monoclonal antibodies (mAb) are an effective new preventative treatment for episodic and chronic migraine, with a favourable adverse event profile. In addition to its role in mediating migraine attacks, CGRP is widely expressed throughout the body and in general has an antiinflammatory/immunoregulatory role. Thus, inhibitory CGRP may potentiate a pro-inflammatory state.

Methods/Results We present a case series of eight patients with new or worsened inflammatory pathology in close temporal association with the start of CGRP mAb therapy.

Conclusions This case series provides novel insights on the potential molecular mechanisms and side-effect profile of CGRP inhibition and warrants vigilance in clinical practice.

\section{ACUTE MONOPLEGIA}

${ }^{1,2}$ Kristen Lefever, ${ }^{3}$ Jared Eisemann, ${ }^{3}$ Letitia Gore, ${ }^{2}$ Laura Clarke, ${ }^{1,2}$ Cullen O'Gorman, ${ }^{1}$ Andrew Swayne, 'Daniel Schweitzer. 'Neurosciences, Mater Public Hospital, Brisbane, QLD, Australia; ${ }^{2}$ Queensland Health, Woolloongabba, QLD, Australia; Infectious Disease, Mater Hospital, Brisbane, QLD, Australia

\subsection{6/bmino-2021-ANZAN.77}

Objectives Pyomyositis is a purulent infection of skeletal muscle, most commonly resulting from haematogenous spread of Staphylococcus aureus infection in immunocompromised individuals ${ }^{1}$. Early recognition and management is important to prevent dissemination of infection and associated complications, including death.

Methods Single case report.

Results 63year old female represented to the emergency department with a three day history of a painful left arm, radiating from the neck. More recently, she had developed proximal left arm weakness and paraesthesia. She had suffered from a self-resolving diarrhoeal illness in the 48hours prior. Clinical examination confirmed a proximal left arm weakness of lower motor neuron pattern. Initial investigations demonstrated a normal creatinine kinase (CK) and white cell count, but elevated inflammatory markers. MRI cervical spine imaging excluded degenerative disease but raised suspicion of oedema within the left sided posterior cervical musculature. Subsequent blood cultures isolated methicillin sensitive Staphylococcus aureus. The patient developed lower back pain and fevers prompting further imaging, demonstrating multifocal epidural abscesses. There was no evidence of infective endocarditis. She received six weeks of intravenous flucloxacillin, followed by oral therapy, resulting in normalization of her inflammatory markers. Delayed repeat MRI imaging confirmed resolution of the epidural infection and improvement in left shoulder musculature oedema and hyperenhancement, which correlated with marked clinical improvement.

Conclusion Pyomyositis is increasingly recognised in temperate climates and immunocompetent individuals. Pain is an early feature, with CK often remaining normal throughout the disease. ${ }^{1}{ }^{2}$ Pyomyositis should be considered as a differential diagnosis in all patients with new onset painful weakness.

\section{REFERENCES}

1. Crum. Bacterial pyomyositis in the United States. The American Journal of Medicine 2004:117:420-428.

2. Bickels, Ben-Sira, Kessler, Wientroub. Primary pyomyositis. The Journal of Bone and Joint Surgery 2002;84:2277-2286.

\section{A CASE OF NON-CONVULSIVE STATUS EPILEPTICUS (NCSE) AS FIRST PRESENTATION OF SPORADIC CREUTZFELDT-JAKOB DISEASE (SCJD)}

${ }^{1}$ Luke Gagen, ${ }^{2,3}$ Kristen Lefever, ${ }^{3}$ Daniel Schweitzer, ${ }^{2}$ Laura Clarke, ${ }^{3}$ Cullen O'Gorman, ${ }^{3}$ Andrew Swayne. 'University of Queensland, Brisbane, QLD, Australia; ${ }^{2}$ Queensland Health, Woolloongabba, QLD, Australia; ${ }^{3}$ Neurosciences, Mater Public Hospital, Brisbane, QLD, Australia

\subsection{6/bmjno-2021-ANZAN.78}

Objectives sCJD is a neurodegenerative prion disease characterised by rapidly progressive neuropsychiatric symptoms and movement disorder. Seizures are an uncommon first presentation of sCJD. We present a case of a 71 year-old female in whom presented in NCSE.

Methods Single Case Report.

Results A 71-year-old female initially presented with higher cortical impairment characterised by visuospatial deficits and apraxia suggestive of parietal lobe dysfunction. Subsequent MRI showed multifocal patches of gyriform diffusion restriction - cortical ribboning - predominantly in the right parietal cortex. Autoimmune and metabolic cerebrospinal fluid (CSF) testing were unremarkable for markers of autoimmune or infectious encephalitis. EEG demonstrated NCSE arising from this right hemisphere lesion, with severe diffuse encephalopathy. A ward-based anti-epileptic drug regime was instituted without success. ICU management with midazolam infusion and intubation for NCSE was required. Clinically and electrographically the patient improved over a two-week period, with resolution of NCSE on repeat EEG. Unfortunately however a repeat MRI on Day 17 of admission demonstrated progression of cortical ribboning beyond the initial focus across hemispheres. This in combination with a positive 14-3-3 and tau protein on CSF, raised the likelihood of sCJD. Marked clinical deterioration followed extubation, with palliative care involvement and end of life planning. The diagnosis of sCJD was confirmed with brain biopsy at autopsy.

Conclusion The case is notable as it is rare for sCJD to present early with $\mathrm{NCSE}^{1}$ and reinforces the need for atypical presentations to prompt exploration of a broader differential diagnosis in the context of unexpected investigation findings.

\section{REFERENCE}

1. Espinosa PS, Bensalem-Owen MK, Fee DB. Sporadic creutzfeldt-jakob disease presenting as nonconvulsive status epilepticus case report and review of the literature. Clinical Neurology and Neurosurgery 2010 July 1;112(6):537-40. 